\title{
Use of machine learning techniques to identify HIV predictors for screening in sub-Saharan Africa
}

Charles K. Mutai ${ }^{*}$, Patrick E. McSharry ${ }^{1,2,3}$, Innocent Ngaruye ${ }^{4}$ and Edouard Musabanganji ${ }^{5}$

\begin{abstract}
Aim: HIV prevention measures in sub-Saharan Africa are still short of attaining the UNAIDS 90-90-90 fast track targets set in 2014. Identifying predictors for HIV status may facilitate targeted screening interventions that improve health care. We aimed at identifying HIV predictors as well as predicting persons at high risk of the infection.

Method: We applied machine learning approaches for building models using population-based HIV Impact Assessment (PHIA) data for 41,939 male and 45,105 female respondents with 30 and 40 variables respectively from four countries in sub-Saharan countries. We trained and validated the algorithms on $80 \%$ of the data and tested on the remaining 20\% where we rotated around the left-out country. An algorithm with the best mean $\mathrm{f} 1$ score was retained and trained on the most predictive variables. We used the model to identify people living with HIV and individuals with a higher likelihood of contracting the disease.
\end{abstract}

Results: Application of XGBoost algorithm appeared to significantly improve identification of HIV positivity over the other five algorithms by $\mathrm{f} 1$ scoring mean of $90 \%$ and $92 \%$ for males and females respectively. Amongst the eight most predictor features in both sexes were: age, relationship with family head, the highest level of education, highest grade at that school level, work for payment, avoiding pregnancy, age at the first experience of sex, and wealth quintile. Model performance using these variables increased significantly compared to having all the variables included. We identified five males and 19 females individuals that would require testing to find one HIV positive individual. We also predicted that $4.14 \%$ of males and $10.81 \%$ of females are at high risk of infection.

Conclusion: Our findings provide a potential use of the XGBoost algorithm with socio-behavioural-driven data at substantially identifying HIV predictors and predicting individuals at high risk of infection for targeted screening.

Keywords: Socio-behavioral, Screening, High-risk, Predictors, XGBoost

\section{Background literature}

HIV continues to be significantly the most threatening infectious disease and a burden to public health globally. In the year 2019, global estimates show that 38 million people are living with HIV while 1.7 million and 690,000 thousand are reported new cases and deaths

\footnotetext{
*Correspondence: charlimtai@gmail.com

${ }^{1}$ African Center of Excellence in Data Science, University of Rwanda,

Kigali BP 4285, Rwanda

Full list of author information is available at the end of the article
}

respectively, despite the remarkable progress in diagnosis and access to antiretroviral therapy (ART) [1]. More than half of people living with HIV, $42.9 \%$ of new infections, and $43.5 \%$ of deaths due to AIDS are concentrated in East and Southern Africa [1]. In 2018, 1.6 million, 1 million, 210,000 thousands and 1.2 million people were living with HIV, 72,000, 38,000, 7,800 and 48,000 were newly infected people and 24,000, 13,000, 2,400 and 17,000 deaths were from AIDS-related illness in Tanzania, Malawi, Eswatini and Zambia respectively [2]. The 
Joint United Nations Programme (UNAIDS) had set goals towards stopping AIDS as a public health threat by 2030 [3, 4]. However, the COVID-19 pandemic is already thwarting the progress made, and it can adversely lead to additional AIDS-related deaths in sub-Saharan Africa by the end of 2021 [5, 6].

Despite universal HIV intervention efforts in East and Southern Africa, the geographical distribution of the HIV epidemic is still widely varied $[7,8]$. The region being a resource constraint can not have every intervention for everyone and everywhere. Granular information concerning the HIV epidemic needs tailor-made solutions to address and help protect specific individuals [9]. To identify the most vulnerable individuals for the infection globally, strategies are geared towards optimal allocation of resources and thus higher impact and efficiency contrary to a homogeneous distribution of resources $[10,11]$. Behavioural and social-demographic factors are among significant contributions of HIV transmission and require investigation on the nature of the impact on the HIV epidemic in a particular population [12]. Despite HIV screening being an effective method of identifying individual status, it has challenges and constraints [13]. Community-based HIV screening has successfully improved the identification of people living with HIV [14]. One of the ways of diagnosing people living with HIV is through universal screening of individuals attending health care facilities, but this can be costly for the low-risk population compared to selective testing of those at high risk [15]. Including social-demographic factors in the analysis may extensively improve the potential of predicting those at higher risk of the infection, enhancing optimal choices in the screening process, and helping to facilitate testing and counselling for HIV [16]. This may also disclose individuals who may need PrEP, among other necessary early interventions [17, 18].

Machine learning entails the utilisation of computational and statistical algorithms to determine hidden associations of data that might increase predictions through relaxation of the modelling postulates advanced by standard approaches [19]. Among the recent advances in prediction tools and identification techniques in HIV statistical data [20-22], machine learning offers greater capability in processing huge amounts of data. Its recent application in the identification of potential candidates for preexposure prophylaxis (PrEP) in the USA and Denmark and a population-based research setting in Eastern Africa highlights some of its capabilities [23]. Klon et al. used Laplacian-modified naïve Bayesian to identify active inhibitor compounds from a target database [24]. Another example is the use of electronic health record data in developing HIV prediction models for identifying PrEP candidates in an extensive healthcare system [25]. A machine learning algorithm has been developed that can automatically select important HIV risk-related variables using patients' demographic and clinical data [26].

A review of the use of machine learning approaches in studying HIV/AIDS infection was previously published [27]. The paper by Lee et al. used machine learning approaches in classifying patients with and without the toxicity of biomarkers of mitochondrial in HIV [28]. Recently, Orel et al. used machine learning techniques on the Demographic Health Survey of 10 countries to identify HIV Positive individuals [29].

This paper aims at using machine learning algorithms to identify the HIV predictors of persons using sociobehavioural features and predict those at increased risk of infection in the East and Southern African countries.

\section{Methods \\ Data}

We used the Population-based HIV Impact Assessment (PHIA) project that consists of cross-sectional household-based surveys designed to assess HIV-related key health indicators [30]. ICAP, based in Columbia University in collaboration with the US Centers for Disease Control and Prevention (CDC) and the ministries of Health, manages and implements the PHIA project. The PHIA project is assessing programs of HIV in countries supported by the President's Emergency Plan for AIDS Relief (PEPFAR) by national household surveys.

It was established in 2015 and geared towards documenting the achievements of HIV programs in participating countries as well as ensuring a better understanding of the regional burden trends of the disease in developing countries. PHIA conducts surveys in 14 countries: Côte d'Ivoire, Cameroon, Ethiopia, Eswatini, Haiti, Kenya, Lesotho, Zimbabwe, Malawi, Namibia, Rwanda, Tanzania, Uganda and Zambia. More details on the PHIA survey have been reported elsewhere [31].

We only included individuals tested for HIV in our analysis from the recently released PHIA survey data for Tanzania (2016-2017), Zambia (2016), Malawi (20152016) and Eswatini (2016-2017). Countries whose data were not yet released were excluded from the study. We merged adult datasets and HIV test results from the four countries to obtain two sets of data, comprising 41,939 male and 45,105 female respondents with $8.5 \%$ and $13.3 \%$ HIV positive cases respectively, Table 1 . Background characteristics of the dataset are displayed in table A3. We considered two HIV test outcomes for respondents, positive and negative, thereby requiring the construction of a binary classifier using machine learning. 
Table 1 Summary of Phia dataset

\begin{tabular}{lllll}
\hline Characteristics & Levels & Overall & HIV Positive & HIV Negative \\
\hline $\begin{array}{l}\text { n (Total number of individu- } \\
\text { als,\%) }\end{array}$ & 87,044 & $9,533(11.0)$ & $77,511(89.0)$ \\
Gender, n (\%) & Males & 41,939 & $3,552(8.5)$ & $38,387(91.5)$ \\
& Females & 45,105 & $5,981(13.3)$ & $39,124(86.7)$ \\
Country, n (\%) & Malawi & 19,829 & $2,100(10.6)$ & $17,729(89.4)$ \\
& Eswatini & 11,875 & $3,230(27.2)$ & $8,645(72.8)$ \\
& Zambia & 21,280 & $2,569(12.1)$ & $18,711(87.9)$ \\
& Tanzania & 34,060 & $1,634(4.8)$ & $32,426(95.2)$ \\
\hline
\end{tabular}

\section{Data pre-processing}

We pooled datasets from the four countries and merged HIV test results with the adult interview datasets. We then resampled the data utilising sample weights of HIV test outcomes per country thus compensating for noncoverage, non-response and population total adjustment weights. Then, we joined the data sets from the four countries into one data frame with 238 variables each for both sexes. We removed variables with more than $30 \%$ missing values, those with no variance, non-unique columns, above $80 \%$ correlated features, indeterminate and non-informative features such as household-id, personid, line-number and others, Table A1. We also encoded both the nominal and ordinal variables using the labelcode and one-hot encode methods appropriately based on the information from the survey [32]. Multiple imputations with chained equations (MICE) [33] was utilized in imputing the missing values in each of these categories. Finally, we further harmonised and scaled the data by standardizing to ensure a fair penalisation of the scheme used for all the regressors, Fig. 1, step 1. This resulted in 41,939 males and 45,105 females in the final dataset corresponding to 26 and 36 variables respectively as shown in, Table A2. From this final dataset, 25 variables of the total variables were similar for both sexes.

\section{Model validation}

In this study, our machine learning task was structured to solve a binary classification problem.

Our dataset comprises healthy individuals labelled negative in one class while the infected individuals are labelled positive in the other class.

We left out one country for later testing and this was rotated around for testing the generalisation of the models separately for males and females. $80 \%$ of the datasets were selected for training while $20 \%$ were used as test samples, Fig. 1, step 1. We chose an 80:20 ratio for our study and it has been shown to achieve the best results among other ratios elsewhere [34]. We randomly picked from a grid 50 sets of control values of the learning process (hyperparameters), and these were used in training and validation of data using each of Elastic Net (EN) [35], k-Nearest Neighbors (KNN) [36], RandomForest (RF) [37], Support Vector Machine (SVM) [38], XGBoost [39] and Light Gradient Boosting (LGBT) [40] algorithms, (Fig. 1, step 2 and 3).

We determined the average scores of $\mathrm{f} 1$ for each of these 50 sets with a five-fold cross-validation plan over the validated samples and the most powerful set of hyperparameters were picked, Fig. 1, step 2. f1 score is a metric that is the most-used member of the parametric family of the $f$-measures, named after the parameter value $\beta=1$, where beta is a factor of recall importance than precision [41]. It is defined as the harmonic mean of precision and recall.

f1 score $=2 *($ Recall $*$ Precision $) /($ Recall + Precision $)$

Precision is the ratio of correctly predicted positive observations to the total predicted positive observations and recall (sensitivity) is the ratio of correctly predicted positive observations to all observations in the actual class. It accounts for both false positives and false negatives and it cannot be influenced by the uneven class distribution and therefore preferred over the accuracy metric [42]. Importantly, the metric used was very sensitive and high yielding in predicting the number of HIV positive persons (precision). We computed the Precision-Recall for our preferred model per sex, displaying the precision for different sensitivities. This curve is not affected by imbalanced datasets and hence preferred over the ROC curve [43].

\section{Model and variable selection with the direction of the association}

The algorithm with the best $\mathrm{f} 1$ score was used in the subsequent analysis where all countries were included. We used the algorithm along with 250 sets of parameters random search in training and validation with a five-fold cross-validation plan. First, we estimated and compared sensitivity, f1 score and positive predictive value results on all the variables. We then conducted a sequential forward floating selection (SFFS) procedure in determining the saturation point of variables based on f1 scoring with $80 \%$ training samples and variables whose f1 scores plateaued from the saturated point were selected. We also evaluated the association of the selected features with the probability of being HIV positive using SHapley Additive exPlanations (SHAP) [44].

SHAP is an important technique that is used in explaining the contribution of each feature in prediction. 


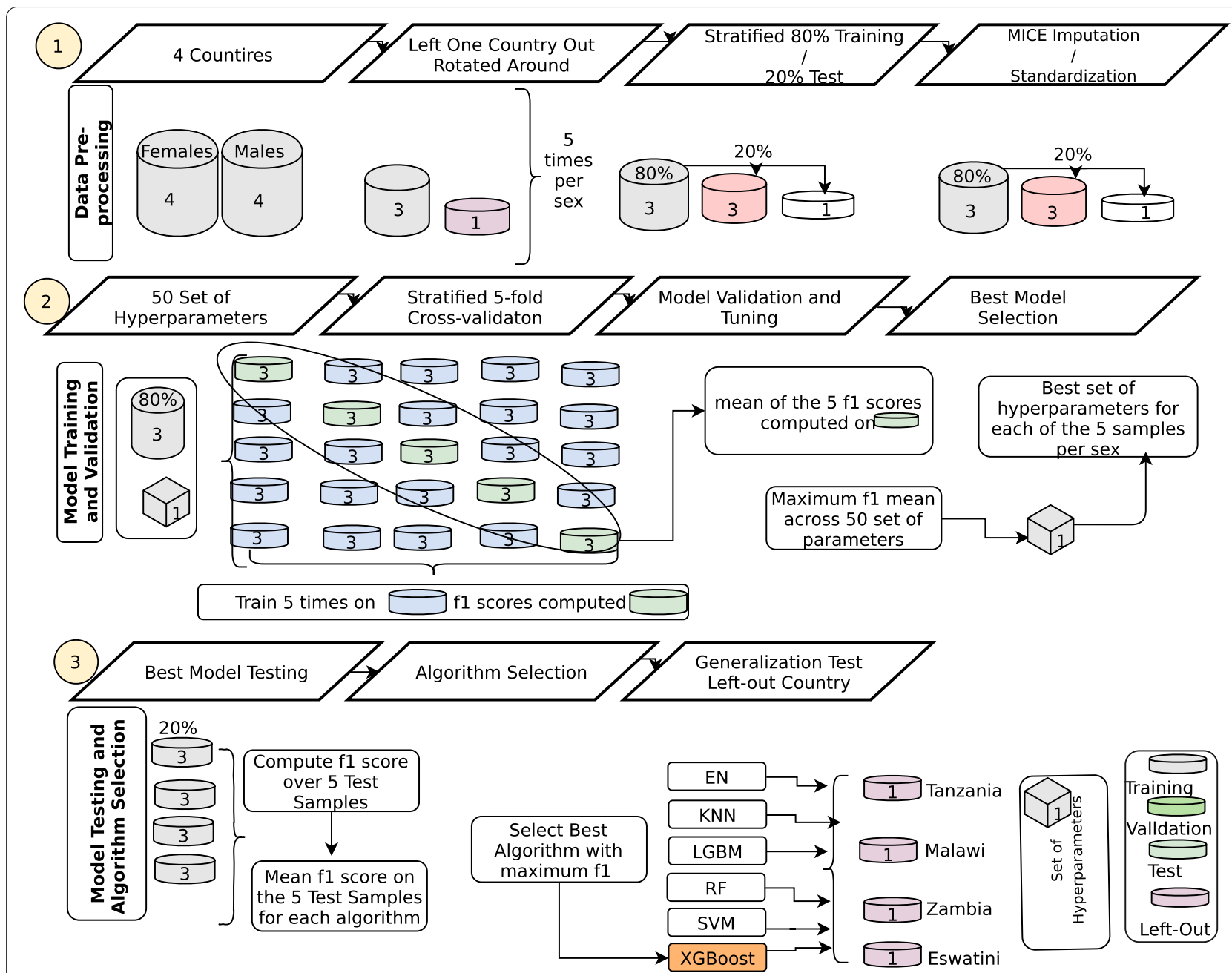

Fig. 1 Diagram explaining the method process

To determine a $95 \%$ sensitivity of individuals living with HIV knowing their status and a 95\% or more probability of individuals being HIV positive we utilized our preferred model to calibrate the given two situations. First, a 95\% sensitivity was set equivalent to $95 \%$ of persons knowing their status and chose a threshold that corresponds to this sensitivity, reporting precision and number of individuals to be tested. Secondly, a population for which the predicted probability of being HIV positive was $95 \%$ or higher was identified. These individuals were considered as either positive targets for testing strategies or HIV negative individuals for prevention aid.

\section{Results}

Overall, there was a varied HIV prevalence ranging from $4.8 \%$ in Tanzania to $27.3 \%$ in Eswatini. The overall HIV positivity was $8.5 \%$ for male and $13.3 \%$ for females, Table 1 . Persons aged 35 years and above displayed a higher HIV prevalence than the younger population in both sexes. In urban regions, $51.1 \%$ of females have HIV, compared to $48.8 \%$ who do not. Similarly, $49.2 \%$ of males in urban areas have HIV, compared to $47.0 \%$ who do not. In the wealth quintiles of males, $32.3 \%$ of the more wealthy persons have HIV, compared to $30.75 \%$ of those who do not. In the wealth quintiles of females, $29.8 \%$ of more wealthy individuals have HIV, compared to $30.9 \%$ of those who do not. $51.0 \%$ of males with the lowest level 
of education have HIV, compared to $549 \%$ who do not. Again, $61.4 \%$ of females with the lowest education level have HIV, compared to $59.9 \%$ who do not, Table A3.

\section{Algorithms results in test samples}

Figure 2, shows that the XGBoost algorithm achieved the highest $\mathrm{f} 1$ score of $90 \%$ and $92 \%$ for males and females respectively, among the six algorithms that were used on all 8 ( 4 per sex) test samples. This was followed closely by the RF algorithm with a score of $87 \%$ for males and $93 \%$ for females. EN algorithm performance was $84 \%$ and $90 \%$ for males and females respectively. SVM performance was $87 \%$ for males and $89 \%$ for females. The LGBM f1 score was $86 \%$ for males and $88 \%$ for females while KNN performed dismally with an $\mathrm{f} 1$ score of $85 \%$ for males and $88 \%$ for females, Table 2 .

\section{Algorithms results in left-out samples}

Similarly, the six algorithms were trained on all four leftout samples. The f1 scores between males and females substantially varied all the algorithms, Fig. 3. However, the XGBoost algorithm got the highest f1 score of $83 \%$ and $85 \%$ for males and females respectively, among the six algorithms. This was followed closely by the LGBM algorithm with a score of $81 \%$ for males and $87 \%$ for females. SVM algorithm performance was $79 \%$ and $86 \%$

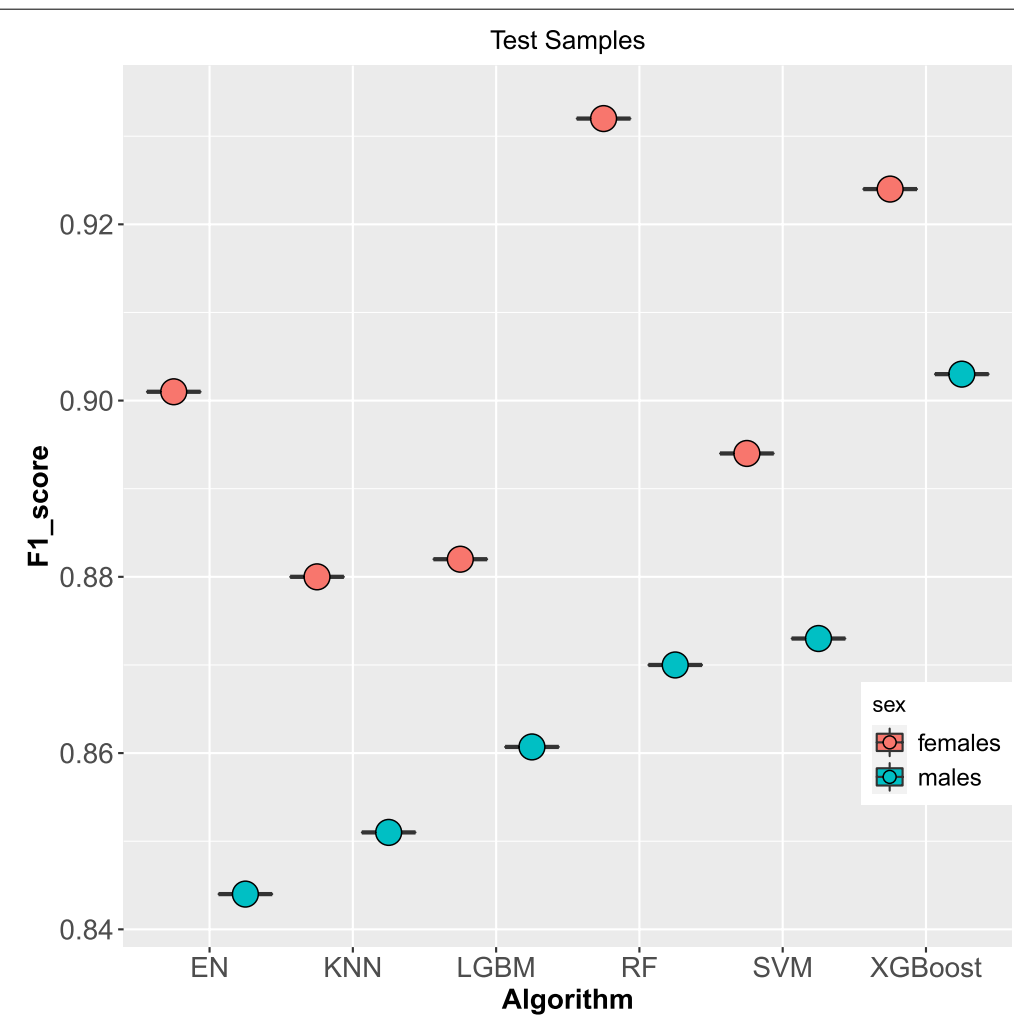

Fig. 2 f1 scores Boxplot on methods used on test samples per sex

Table 2 F1 score for Algorithms on the test, left-out and train samples

\begin{tabular}{|c|c|c|c|c|c|c|}
\hline samples & XGBoost & KNN & SVM & $\mathrm{RF}$ & EN & LGBM \\
\hline males test & 0.90 & 0.85 & 0.87 & 0.87 & 0.84 & 0.86 \\
\hline females test & 0.92 & 0.88 & 0.89 & 0.89 & 0.90 & 0.88 \\
\hline males left-out & 0.83 & 0.81 & 0.79 & 0.79 & 0.72 & 0.81 \\
\hline females left-out & 0.85 & 0.85 & 0.86 & 0.86 & 0.76 & 0.87 \\
\hline males train & 0.90 & 0.85 & 0.86 & 0.91 & 0.83 & 0.86 \\
\hline females train & 0.91 & 0.87 & 0.89 & 0.92 & 0.88 & 0.88 \\
\hline
\end{tabular}




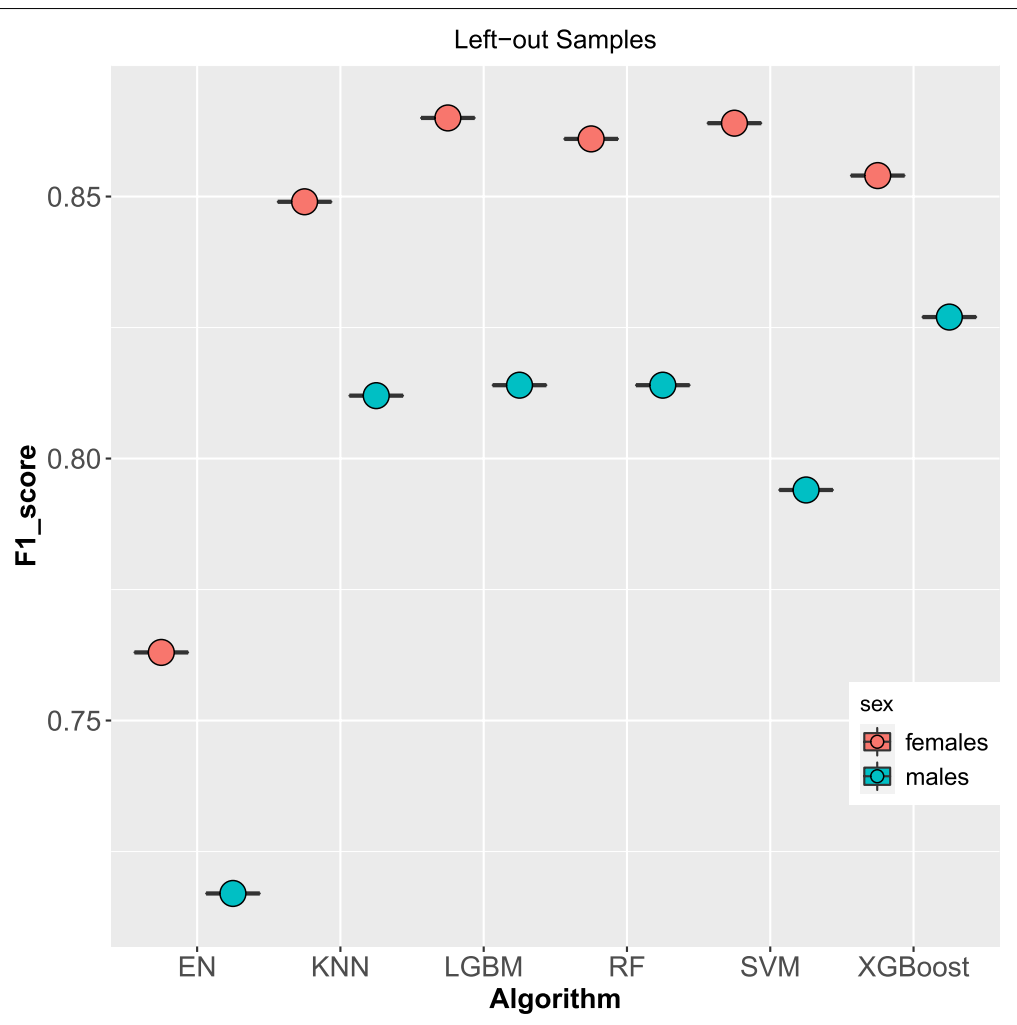

Fig. 3 f1 scores Boxplot on methods used on left-out samples per sex

for males and females respectively. KNN secured a score of $85 \%$ for females and a low score of $81 \%$ for males. RF scored $86 \%$ for females and $81 \%$ for males while EN was the worst-performing algorithm with an $\mathrm{f} 1$ score of $76 \%$ for females and $72 \%$ for males, Table 2.

\section{Variable selection and direction of associations}

SFFS procedure was used in determining the saturation limit, selecting variables based on $\mathrm{f} 1$ scoring. As a result, 15 and 12 most influential features of males and females were selected respectively, Fig. 4 and 5 . To understand how a feature contributes to the output of the model, we plot SHAP values, Fig. 6 and 7 for males and females respectively. These variables are displayed after ranking in descending order, bearing the highest average or median values of Shapley at the top.

Here, all the values on the left represent the observations that shift the predicted value in the negative direction while the points on the right contribute to shifting the prediction in a positive direction. The graph summarises the impact of explanatory features on the model output. Features that increase or decrease the risk of HIV infection are coded in red and blue respectively. Being older, never attending school, at the highest level of education, at the highest grade a school level, in avoidance of pregnancy, in TB treatment, in use alcohol drink, an urban dweller, aware of HIV status, wealthy, nonmarital and circumcised is predictive of HIV positivity.

To determine a 95\% sensitivity of individuals living with HIV knowing their status and a $95 \%$ or more probability of individuals being HIV positive we utilized the XGBoost model to calibrate the given situations $\mathrm{A}$ and $B$ respectively.

\section{Situations}

\section{5\% of individuals living with HIV know their state}

Table 3, shows confusion matrices on test samples. A sensitivity of $95 \%$ for males would need 4,154 individuals out of $8,388(49.52 \%)$ tested to identify 651 HIV positives from 685 persons living with HIV. Correspondingly, therefore, five individuals would need testing to find one person who is HIV positive with a precision of $15.67 \%$. 3,301 individuals out of 9,021 (36.59\%) of females would require testing to detect 1,115 HIV positives out of the 1,173 persons living with HIV. Similarly, the precision is $33.77 \%$ and needs 19 individuals tested. 


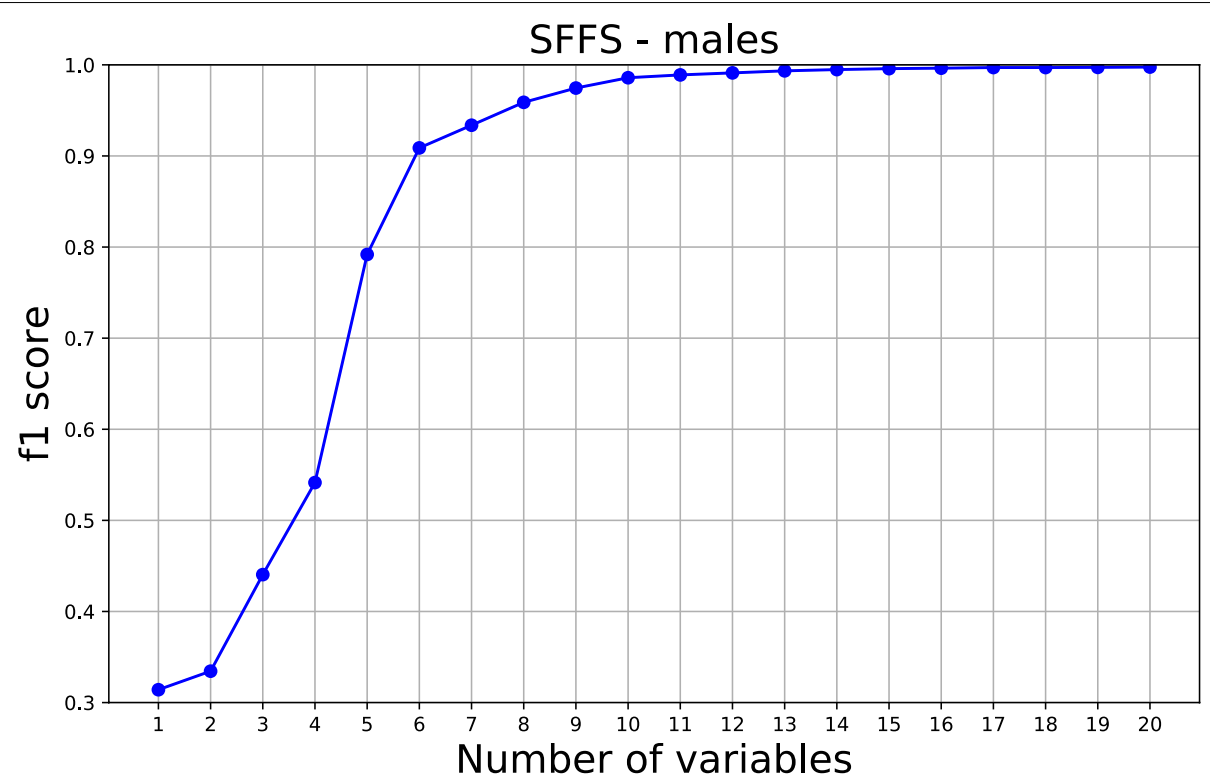

Fig. 4 Sequential floating forward selection (SFFS) for males

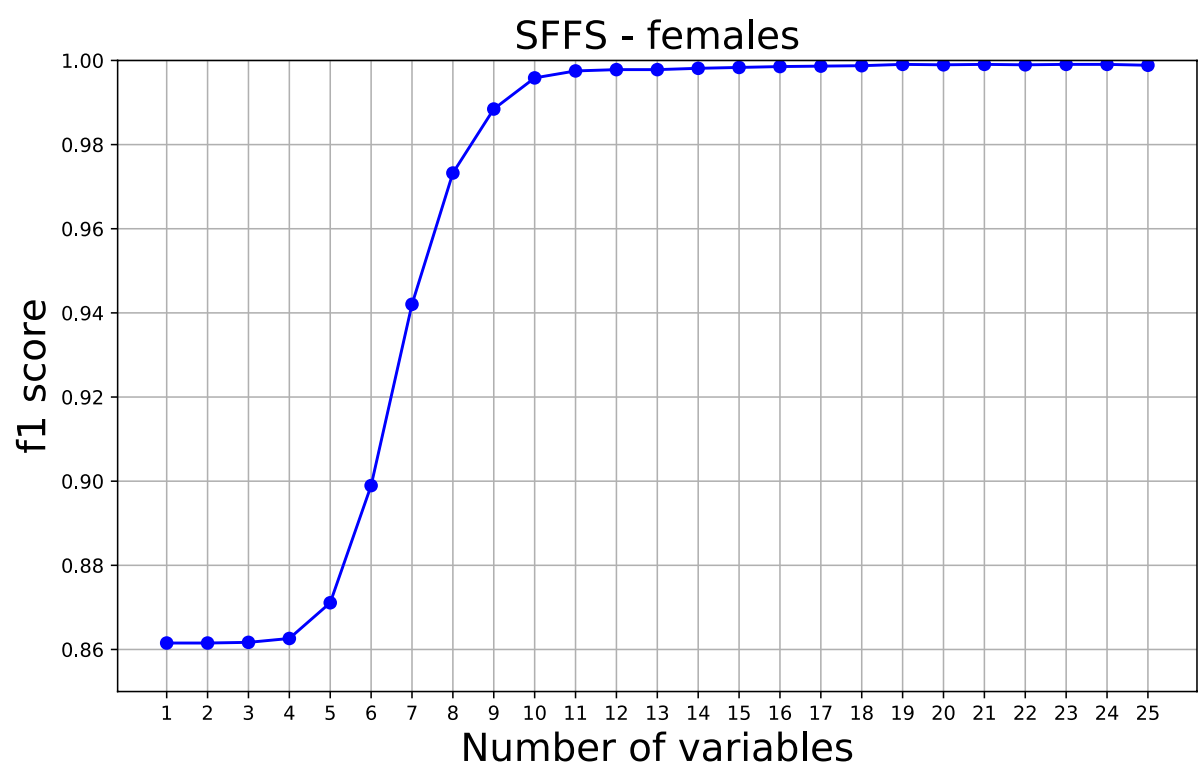

Fig. 5 Sequential floating forward selection (SFFS) for females

\section{5\% or higher probability of being HIV positive}

We identified $348(4 \cdot 14 \%)$ males out of the 8,388 and $975(10.81 \%)$ females out of 9,021 as a high-risk population. We find that 350 males would have been identified HIV positives out of 685 people living with HIV while 969 females would have been correctly identified HIV positives from 1,173 individuals, Table 3. 


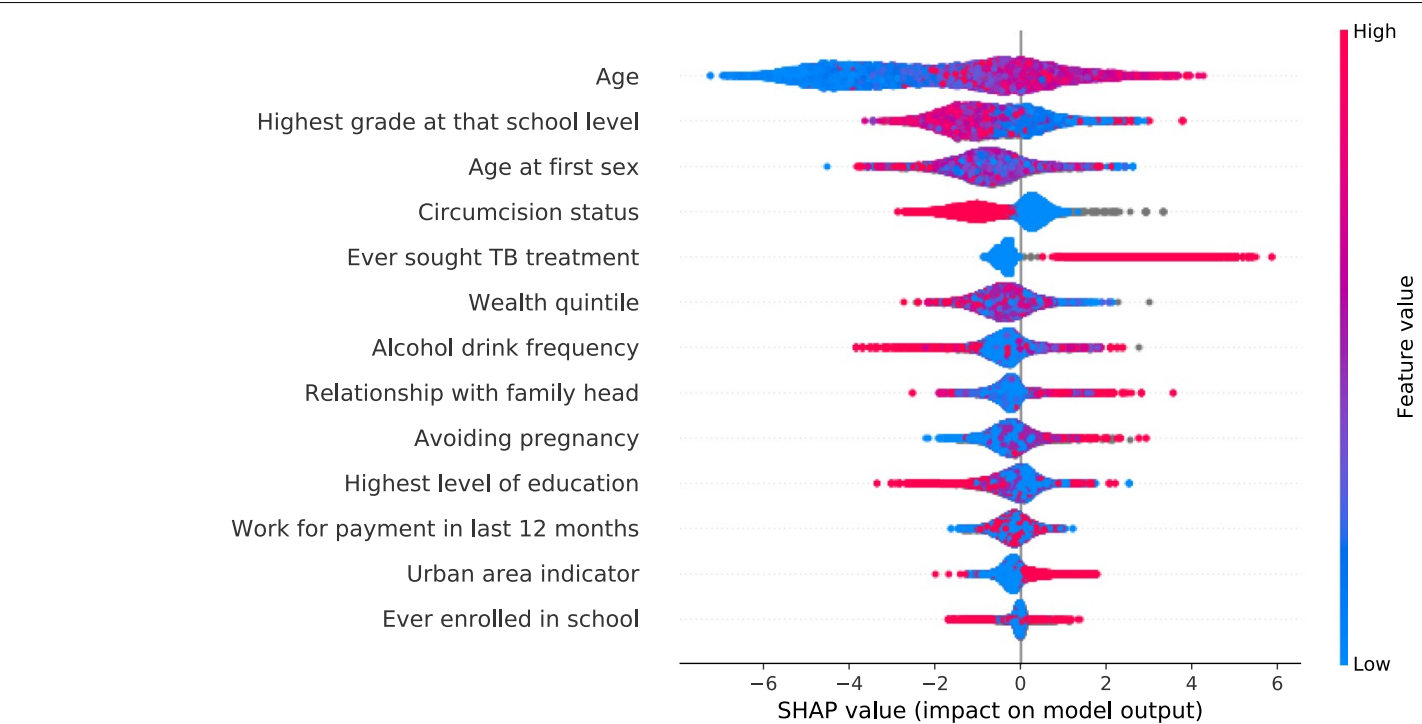

Fig. 6 SHAP summary plots for HIV status predictors in male individuals

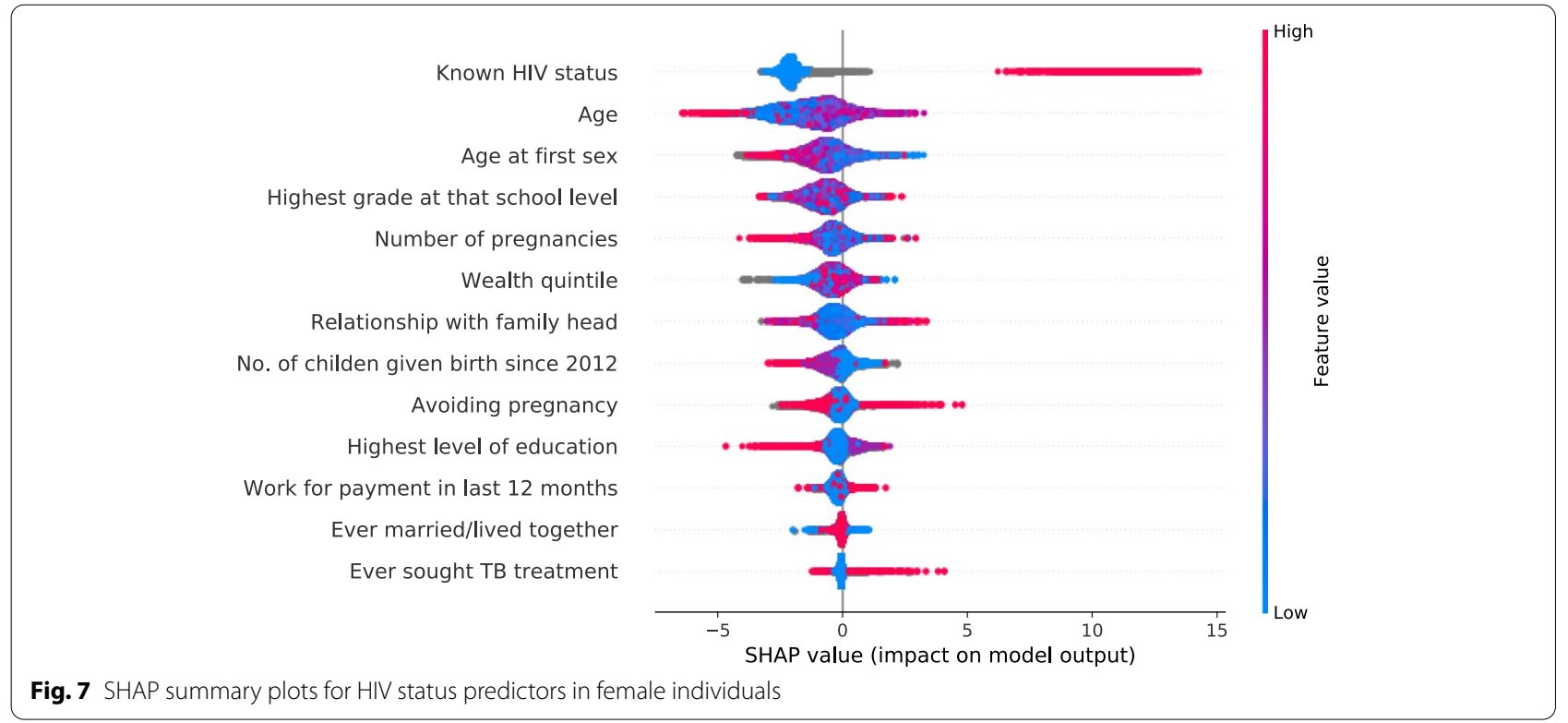

Table 3 People living with HIV know their status and 95\% or more probability of being HIV positive

\begin{tabular}{llllll}
\hline 95\% of those with HIV & TP & FP & FN & TN & precision \\
\hline Know their status (males) & 4200 & 3503 & 34 & 651 & 15.67 \\
Know their status (females) & 5662 & 2186 & 58 & 1115 & 33.77 \\
$\begin{array}{l}\text { 95\% or > probability of } \\
\text { being HIV positive (males) }\end{array}$ & 7690 & 13 & 350 & 335 & 99.26 \\
$\begin{array}{l}\text { 95\% or more probability of } \\
\text { being HIV positive (females) }\end{array}$ & 7842 & 6 & 204 & 969 & 96.26 \\
\hline
\end{tabular}

\section{Discussion}

We used a large dataset of over 80,000 respondents in four countries from the East and Southern Africa region to predict the HIV status of persons by use of sociodemographic factors. We used the XGBoost method in the identification of the most predictive factors of HIV positivity, which delivered better results than the other five algorithms with f1 scores on the sample test of $90 \%$ and $92 \%$ when all variables are included in males and females respectively. 
The method enabled us to establish the most predictive features for HIV status in both sexes: age, relationship with the head of the family, ever enrolled in school, the highest level of education, highest grade at that school level, work for payment in the last 12 months, wether avoiding pregnancy, age at first sex experienced, ever sought TB treatment, frequency of alcohol drinking, urban area indicator, wealth quintile, number of pregnancies, number of births since 2012, marital status and circumcision status.

The course of the association between predictor features and HIV status of individuals was determined through the use of XGBoost along with SHAP plots and illustrated specific feature importance to give an intuitive understanding of the key features. The age of an individual has the highest overall impact on HIV status than other features, and any change of age can have a more remarkable influence than others. More aged individuals have a higher probability of infection in both sexes. Several of those avoiding pregnancy by various methods stand a higher chance of contracting the disease in both sexes. Potential reasons for this may be an increased exposure to sex making them more vulnerable. The majority of those living in urban regions seemed to be more exposed to the disease than their counterparts living in rural areas. Those with a little level of education have low knowledge of mitigation measures of HIV risk and pose a greater risk of HIV in both sexes. HIV positivity is associated with a higher number of those seeking TB treatment; a low CD4 count from HIV patients are at a much higher risk of falling ill from TB infection than those who are negative. Similarly being uncircumcised exposes males to the disease which is consistent with studies by [45] while in females alone, being exposed to sex at an older age, attaining higher grade at school, an increase in the number of children born may lead to a reduction of HIV positivity. Our results are consistent with those of [46] that indicated literacy and urbanity as strong predictors of HIV acquisition and Sing et al., which found that urban dwellers may increase HIV positivity through more contact with high-risk sexual individuals than rural residents [47]. Age, a little level of education and gender being predictors of the disease assert the findings in $[48,49]$.

A $95 \%$ sensitivity was required in ensuring that $95 \%$ of individuals living with HIV knew their status. With the XGBoost algorithm, we utilized 15 and 12 most predictive variables of males and females accordingly to establish 5 and 19, the number required to screen to know one individual with HIV in males and females respectively. These are within the range of 3 to 86 and 4 to 154 for community-based and facility-based screening respectively given in previous studies [50]. We identified $4 \cdot 14 \%$ males and $10.81 \%$ females as a high-risk population in the second situation, which is consistent with previous studies that indicated that about seven women get new infections with HIV for every four men infected [51, 52]. In general, female performance in all our algorithms was slightly higher than those of males in this study. Our method borrows heavily from Orel's approach in predictor identification, and they both choose the Xgboost model algorithm as the best performing model among the alternatives. Our results, on the other hand, show different predictors than those found in Orel's research, with the exception that they both found individual age and wealth as predictors of the disease [29].

Other screening methods exist, but they are not without drawbacks. Universal screening, in which patients are given tests on health care facilities, is limited to a poor cost-benefit ratio in low HIV incidence situations [53]. While indicator-condition-guided testing based on specific medical conditions ignores factors such as age, sex, and medical conditions, which have all been linked to a lower risk of HIV transmission [54]. In generalized epidemic settings, a focus on well-known risk groups such as serodiscordant spouses and young women can effectively reach high-risk individuals [55] but may overlook less well-known or easily defined subgroups at risk [56], resulting in inefficient resource allocation [57]. Despite the absence of a recognized risk factor, self-assessment is one method of recognizing individuals at high risk. However, an individual's risk perception is influenced by their HIV-related awareness, and unanticipated or uncontrolled exposures can go undetected [58]. According to WHO guidelines, PrEP should be targeted at subpopulations considered to be at high risk of HIV infection [59]. However, in the context of a widespread outbreak, the best demographic subgroups to target may not be obvious, and merely providing PrEP to established high-risk subgroups like young people or mobile populations may be ineffective. As a result, a PrEP technique based on more subtle use of individual characteristics may be able to reduce the cost of preventing a new HIV infection. Our method provides an alternative to some of the drawbacks listed above, as well as a potential complementary method for identifying people who may gain most from enhanced mitigation strategies. One limitation of this study is the validity of our model. There was a high degree of missingness and inconclusiveness from selfreported data that potentially impacted the training data.

Our conclusion might reveal the social-behavioural identification of HIV and can enhance screening approaches in limited resources situations. There is a need to adapt HIV screening strategies that better target the adult population, those using contraceptives, urban dwellers, the little educated population, TB 
patients and uncircumcised men. There is an increased number of available surveys with individual-level data that is rich in demographic characteristics, social history, laboratory tests and results of various diseases. More advanced approaches to utilize them can effectively assist in preventing, diagnosing and testing HIV and other diseases. Community-based or facility-based testing programs could incorporate this approach in practice to identify high-risk individuals. However, additional studies are needed to further optimize this model, integrate and apply them into a real-world primary care setting. This may also disclose individuals who may need PrEP, among other risk reduction strategies.

\begin{abstract}
Abbreviations
UNAIDS: The Joint United Nations Programme; HIV: Human Immunodeficiency Virus; AIDS: Acquired Immune Deficiency Syndrome; PHIA: Populationbased HIV Impact Assessment; DHS: Demographic and Health Surveys; ART : Antiretroviral therapy; UNAIDS: The Joint United Nations Programme; PrEP: Pre-exposure prophylaxis; CDC: Centers for Disease Control and Prevention; PEPFAR: President's Emergency Plan for AIDS Relief; EN: Elastic Net; KNN: K-Nearest Neighbors; RF: RandomForest; SVM: Support Vector Machine; LGBT: Light Gradient Boosting; MICE: Multiple imputations with chained equations; SFFS: Sequential forward floating selection; SHAP: SHapley Additive exPlanations.
\end{abstract}

\section{Supplementary Information}

The online version contains supplementary material available at https://doi. org/10.1186/s12874-021-01346-2.

\section{Additional file 1:}

\section{Acknowledgements}

Not applicable.

\section{Authors' contributions}

IN had full access to all of the data in the study and took responsibility for the data integrity. CM conceptualised the idea, processed data into the software and delivered the results for this manuscript. EM ensured the accuracy of the data analysis. IM contributed to the interpretation of the results. CM drafted the initial manuscript as all authors made substantial revisions. PM and EM commented on the final draft of the manuscript while CM finalised the text. All authors read and approved the final manuscript.

\section{Funding}

Not applicable.

\section{Availability of data and materials}

The datasets used and/or analysed during the current study are available from the corresponding author on reasonable request. The code used in the analysis is publicly available on Github (https://github.com/charlesmutai/ solid-disco)

\section{Declarations}

Ethics approval and consent to participate

Not applicable.

\section{Consent for publication}

Not applicable.

\section{Competing interests}

The authors declare that they have no competing interests. All authors approved the final manuscript.

\section{Author details}

${ }^{1}$ African Center of Excellence in Data Science, University of Rwanda, Kigali BP 4285, Rwanda. ${ }^{2}$ College of Engineering, Carnegie Mellon University Africa, Kigali BP 6150, Rwanda. ${ }^{3}$ Oxford Man Institute of Quantitative Finance, Oxford University, Oxford OX2 6ED, UK. ${ }^{4}$ College of Science and Technology, University of Rwanda, Kigali, Rwanda. ${ }^{5}$ College of Business and Economics, University of Rwanda, Kigali, Rwanda.

Received: 30 November 2020 Accepted: 13 July 2021

Published online: 31 July 2021

\section{References}

1. 'UNAIDS data 2020'. https://www.unaids.org/en/resources/documents/ 2020/unaids-data. Accessed 29 Oct 2020.

2. 'Zambia | UNAIDS'. https://www.unaids.org/en/regionscountries/count ries/zambia. Accessed 10 Nov 2020

3. 'Fast-track commitments to end AIDS by 2030 | UNAIDS'. https://www. unaids.org/en/resources/documents/2016/fast-track-commitments. Accessed 30 Nov 2020.

4. '2016 United Nations Political Declaration on Ending AIDS sets world on the Fast-Track to end the epidemic by 2030'. https://www.unaids.org/ en/resources/presscentre/pressreleaseandstatementarchive/2016/june/ 20160608_PS_HLM_PoliticalDeclaration. Accessed 29 Oct 2020.

5. Jewell BL, et al. Potential effects of disruption to HIV programmes in subSaharan Africa caused by COVID-19: results from multiple mathematical models. Lancet HIV. 2020;7(9):e629-40. https://doi.org/10.1016/S23523018(20)30211-3.

6. Dorward J, et al. The impact of the COVID-19 lockdown on HIV care in 65 South African primary care clinics: an interrupted time series analysis. Lancet HIV. 2021;8(3):e158-65. https://doi.org/10.1016/S2352-3018(20) 30359-3.

7. Cuadros DF, et al.,'Mapping the spatial variability of HIV infection in Sub-Saharan Africa: Effective information for localized HIV prevention and control'. Sci Rep. 7, 1, 1, 2017, https://doi.org/10.1038/ s41598-017-09464-y.

8. Zulu LC, Kalipeni E, Johannes E, Analyzing spatial clustering and the spatiotemporal nature and trends of HIV/AIDS prevalence using GIS: the case of Malawi, 1994-2010. BMC Infect Dis. 2014;14(1):285. https://doi.org/10. 1186/1471-2334-14-285.

9. Huerga $\mathrm{H}$, et al. Who Needs to Be Targeted for HIV Testing and Treatment in KwaZulu-Natal? Results From a Population-Based Survey', J. Acquir. Immune Defic Syndr. 2016;73(4):411-8. https://doi.org/10.1097/QAl. 0000000000001081

10. Blower S, Coburn BJ. Maximising the effect of combination HIV prevention in Kenya. Lancet Lond Engl. 2014;384(9952):1426. https://doi.org/10. 1016/S0140-6736(14)61859-6.

11. Aral SO, Torrone E, Bernstein K. Geographical targeting to improve progression through the sexually transmitted infection/HIV treatment continua in different populations. Curr Opin HIV AIDS. 2015;10(6):477-82. https://doi.org/10.1097/COH.0000000000000195.

12. 'Social and Behavioural Aspects of the HIV Epidemic--A Review on JSTOR' https://www.jstor.org/stable/2982186. Accessed 6 Nov 2020.

13. Rizza SA, MacGowan RJ, Purcell DW, Branson BM, Temesgen Z. HIV Screening in the Health Care Setting: Status, Barriers, and Potential Solutions. Mayo Clin Proc. 2012;87(9):915-24. https://doi.org/10.1016/j. mayocp.2012.06.021.

14. Celum C, Barnabas R. Reaching the 90-90-90 target: lessons from HIV self-testing. Lancet HIV. 2019;6(2):e68-9. https://doi.org/10.1016/S23523018(18)30289-3

15. Zheng W, Balzer $L$, van der Laan M, Petersen M. Constrained binary classification using ensemble learning: an application to cost-efficient targeted PrEP strategies. Stat Med. 2018;37(2):261-79. https://doi.org/10. 1002/sim.7296 
16. Obermeyer $\mathrm{CM}$, Osborn $\mathrm{M}$. The utilization of testing and counseling for HIV: a review of the social and behavioral evidence. Am J Public Health. 2007;97(10):1762-74. https://doi.org/10.2105/AJPH.2006.096263.

17. De Cock KM, Barker JL, Baggaley R, El Sadr WM. Where are the positives? HIV testing in sub-Saharan Africa in the era of test and treat', AIDS Lond Engl. 2019;33(2):349-52. https://doi.org/10.1097/QAD.0000000000 002096.

18. Ahmed S, et al. Lost opportunities to identify and treat HIV-positive patients: results from a baseline assessment of provider-initiated HIV testing and counselling (PITC) in Malawi. Trop Med Int Health. 2016;21(4):479-85. https://doi.org/10.1111/tmi.12671.

19. J. Sidey-Gibbons and C. Sidey-Gibbons, 'Machine learning in medicine: a practical introduction', BMC Med Res Methodol. 2019;19. https://doi.org/10. 1186/s12874-019-0681-4.

20. Tang D, et al.,'Application of Data Mining Technology on Surveillance Report Data of HIV/AIDS High-Risk Group in Urumqi from 2009 to 2015', Complexity, 10, 2018. https://www.hindawi.com/journals/complexity/2018/91932 48/. Accessed 17 Feb 2021

21. Hailu T. Comparing Data Mining Techniques in HIVTesting Prediction. Intell Inf Manag. 2015:07:152-79. https://doi.org/10.4236/iim.2015.73014.

22. Agrebi S, Larbi A. Use of artificial intelligence in infectious diseases. Artif Intell Precis Health. 2020:415-38. https://doi.org/10.1016/B978-0-12817133-2.00018-5.

23. Marcus JL, Sewell WC, Balzer LB, Krakower DS. Artificial Intelligence and Machine Learning for HIV Prevention: Emerging Approaches to Ending the Epidemic. Curr HIV/AIDS Rep. 2020;17(3):171-9. https://doi.org/10.1007/ s11904-020-00490-6.

24. Klon AE, Glick M, Davies JW. Application of machine learning to improve the results of high-throughput docking against the HIV-1 protease. J Chem Inf Comput Sci. 2004;44(6):2216-24. https://doi.org/10.1021/ci0497861.

25. Marcus JL, Hurley LB, Krakower DS, Alexeeff S, Silverberg MJ, Volk JE. Use of electronic health record data and machine learning to identify candidates for HIV pre-exposure prophylaxis: a modelling study. Lancet HIV. 2019;6(10):e688-95. https://doi.org/10.1016/S2352-3018(19)30137-7.

26. A. I. L. LearningNeuroscienceNeurotech.July 8 and 2019, 'Two new algorithms can identify patients at risk of HIV.' Neuroscience News. 2019. https:// neurosciencenews.com/hiv-algorithms-14441/. Accessed 2 Nov 2020.

27. Kumari S, Chouhan U, Suryawanshi SK.'Machine learning approaches to study HIV / AIDS infection: A Review', 2012. https://doi.org/10.21786/bbrc/ $10.1 / 6$.

28. Lee JS, Paintsil E, Gopalakrishnan V, Ghebremichael M. 'A comparison of machine learning techniques for classification of HIV patients with antiretroviral therapy-induced mitochondrial toxicity from those without mitochondrialtoxicity. BMC Med Res Methodol. 2019;19(1):216. https://doi. org/10.1186/s12874-019-0848-z.

29. Orel E, Esra R, Estill J, Marchand-Maillet S, Merzouki A, Keiser O.'Machine learning to identify socio-behavioural predictors of HIV positivity in East and Southern Africa', medRxiv. 2020. https://doi.org/10.1101/2020.01.27.20018 242.

30. 'PHIA Project Document Manager - Datasets'. https://phia-data.icap.colum bia.edu/files. Accessed 29 Oct 2020.

31. 'Population-based HIV Impact Assessment (PHIA) Data Use Manual Google Search'. https://www.google.com/search?q=Population-based+ HIV+Impact+Assessment+(PHIA)+Data+Use+Manual+\%E2\%80\%8B\& oq=Population-based +HIV+Impact+Assessment+(PHIA)+Data+Use+ Manual+\%E2\%80\%8B\&aqs=chrome. $69157.585 j 0 j 7 \&$ client=ubuntu\&sourc eid $=$ chrome\&ie $=$ UTF-8. Accessed 29 Oct 2020.

32. Kuhn M, Johnson K. Feature Engineering and Selection: A Practical Approach for Predictive Models, 1st edition. Chapman and Hall/CRC, 2019

33. Buuren S, Groothuis-Oudshoorn C.'MICE: Multivariate Imputation by Chained Equations in R. J Stat Softw. 2011;45. https://doi.org/10.18637/jss. v045.i03.

34. Prashanth DS, Mehta RVK, Sharma N. Classification of Handwritten Devanagari Number - An analysis of Pattern Recognition Tool using Neural Network and CNN. Procedia Comput Sci. 2020;167:2445-57. https://doi.org/ 10.1016/j.procs.2020.03.297.

35. Zou H, Hastie T. 'Regularization and variable selection via the elastic net', JR Stat Soc Ser B Stat Methodol. 2005;67(2):301-20. https://doi.org/10.1111/j. 1467-9868.2005.00503.x.

36. Zhang Z. Introduction to machine learning: k-nearest neighbors. Ann Transl Med. 2016;4:11. https://doi.org/10.21037/atm.2016.03.37.
37. Breiman L. Random Forests. Mach Learn. 2001;45(1):5-32. https://doi.org/10. 1023/A:1010933404324.

38. Cortes C, Vapnik V. Support-vector networks. Mach Learn. 1995;20(3):273-97. https://doi.org/10.1007/BF00994018.

39. Chen T, Guestrin C. 'Xgboost: A scalable tree boosting system,' in Proceedings of the 22nd acm sigkdd international conference on knowledge discovery and data mining, 2016, 785-794.

40. Fan J, Ma X, Wu L, Zhang F, Yu X, Zeng W. Light Gradient Boosting Machine: An efficient soft computing model for estimating daily reference evapotranspiration with local and external meteorological data. Agric Water Manag. 2019;225.

41. 'F-Score', DeepAl, May 17, 2019. https://deepai.org/machine-learning-gloss ary-and-terms/f-score. Accessed 20 May 2021.

42. Goutte C, Gaussier E. 'A Probabilistic Interpretation of Precision. Recall and F-Score, with Implication for Evaluation.' 2005;3408:345-59. https://doi.org/ 10.1007/978-3-540-31865-1_25.

43. Saito T, Rehmsmeier M. 'The Precision-Recall Plot Is More Informative than the ROC Plot When Evaluating Binary Classifiers on Imbalanced Datasets', PLOS ONE. 2015;10(3):e01 18432. https://doi.org/10.1371/journal.pone.0118432.

44. 'A Unified Approach to Interpreting Model Predictions'. https://papers, nips.cc/paper/7062-a-unified-approach-to-interpreting-model-predi ctions. Accessed 2 Nov 2020.

45. Agot KE, Ndinya-Achola JO, Kreiss JK, Weiss NS. Risk of HIV-1 in Rural Kenya: A Comparison of Circumcised and Uncircumcised Men. Epidemiology. 2004;15(2):157-63.

46. Baranczuk Z, et al.,'Socio-behavioural characteristics and HIV: findings from a graphical modelling analysis of 29 sub-Saharan African countries', J. Int. AIDS Soc. 2019;22(12):e25437. https://doi.org/10.1002/jia2.25437.

47. Sing RK, Patra S. 'What Factors are Responsible for Higher Prevalence of HIV Infection among Urban Women than Rural Women in Tanzania?', Ethiop. J Health Sci. 2015;25(4):4. https://doi.org/10.4314/ejhs.v25i4.5.

48. Kharsany ABM, Karim QA. HIV Infection and AIDS in Sub-Saharan Africa: Current Status, Challenges and Opportunities. Open AIDS J. 2016;10:34-48. https://doi.org/10.2174/1874613601610010034.

49. Mondal MNI, Shitan M. 'Factors affecting the HIV/AIDS epidemic: An ecological analysis of global data. Afr Health Sci. 2013;13(2):2. https://doi.org/10. 4314/ahs.v13i2.15.

50. Suthar $A B$, et al.,'Towards Universal Voluntary HIVTesting and Counselling: A Systematic Review and Meta-Analysis of Community-Based Approaches'. PLOS Med. 2013;10(8):e1001496. https://doi.org/10.1371/journal.pmed. 1001496.

51. Dellar RC, Dlamini S, Karim QA. 'Adolescent girls and young women: key populations for HIV epidemic control. J Int AIDS Soc. 2015;18(2 Suppl 1):19408. https://doi.org/10.7448/IAS.18.2.19408.

52. 'Women and girls, HIV and AIDS', Avert, Jul. 20, 2015. https://www.avert.org/ professionals/hiv-social-issues/key-affected-populations/women. Accessed 13 Nov 2020.

53. Yazdanpanah Y, et al. Routine HIV Screening in France: Clinical Impact and Cost-Effectiveness. PLoS ONE. 2010;5.

54. Sullivan A, et al. Feasibility and Effectiveness of Indicator Condition-Guided Testing for HIV: Results from HIDES I (HIV Indicator Diseases across Europe Study). PLOS ONE. 2013;8.

55. 'WHO |WHO expands recommendation on oral pre-exposure prophylaxis of HIV infection (PrEP),'WHO. http://www.who.int/hiv/pub/prep/policybrief-prep-2015/en . Accessed 24 May 2021.

56. Kagaayi J, et al.,'Indices to measure risk of HIV acquisition in Rakai, Uganda', PloS One. 2014;9(4):e92015. https://doi.org/10.1371/journal.pone.0092015.

57. Cambiano V, Miners A, Phillips A. What do we know about the cost-effectiveness of HIV preexposure prophylaxis, and is it affordable? Curr Opin HIV AIDS. 2016;1 (1):56-66. https://doi.org/10.1097/COH.0000000000000217.

58. Maughan-Brown B, Venkataramani AS. 'Accuracy and determinants of perceived HIV risk among young women in Africa. BMC Public Health. 2017;18. https://doi.org/10.1186/s12889-017-4593-0.

59. 'WHO | Guideline on when to start antiretroviral therapy and on pre-exposure prophylaxis for HIV'. https:/www.who.int/hiv/pub/guidelines/early release-arv/en/. Accessed 24 May 2021.

\section{Publisher's Note}

Springer Nature remains neutral with regard to jurisdictional claims in published maps and institutional affiliations. 